\title{
Concise Enantioselective Syntheses of (+)-L-733,060 and (2S,3S)-3-Hydroxy- pipecolic Acid by Cobalt(III)(salen)-Catalyzed Two-Stereocenter Hydrolytic Kinetic Resolution of Racemic Azido Epoxides
}

\author{
Dattatray A. Devalankar, Pandurang V. Chouthaiwale, Arumugam Sudalai* \\ Chemical Engineering \& Process Development Division, CSIR-National Chemical Laboratory, Dr. Homi Bhabha Road, Pune, \\ Maharashtra 411008, India \\ Fax+91(20)25902676; E-mail: a.sudalai@ncl.res.in \\ Received: 21.08.2013; Accepted after revision: 01.10.2013
}

\begin{abstract}
An efficient synthesis of the 2,3-disubstituted piperidines (+)-L-733,060 and (2S,3S)-3-hydroxypipecolic acid $(\geq 99 \%$ ee) in high optical purity from commercially available starting materials is described. The strategy involves a cobalt-catalyzed hydrolytic kinetic resolution of a racemic azido epoxide with two stereocenters and an intramolecular reductive cyclization as key reactions.
\end{abstract}

Key words: azides, epoxides, stereoselective synthesis, Wittig reactions, cyclizations, piperidines

Chiral 2,3-disubstituted piperidine moieties with a $\beta$-hydroxy functional groups are found in numerous natural products and are common subunits in drugs and drug candidates. ${ }^{1}$ Selected examples include (+)-L-733,060 (1) ${ }^{2}$ and (+)-CP-99,994 (2), ${ }^{3}$ both potent and selective nerokinin-1 substance P receptor antagonists; febrifugine (4), ${ }^{4}$ an antimalarial agent; (-)-swainsonine (5), ${ }^{5}$ an inhibitor of lysosomal $\alpha$-mannosidase and a potent anticancer drug; and $(2 S, 3 S)$-3-hydroxypipecolic acid [3; $(2 S, 3 S)$-3-hydroxypiperidine-2-carboxylic acid], ${ }^{6}$ a key precursor in the syntheses of $\mathbf{4}$ and $\mathbf{5}$ (Figure 1).<smiles>FC(F)(F)c1cc(CO[C@H]2CCCN[C@H]2c2ccccc2)cc(C(F)(F)F)c1</smiles><smiles>COc1ccccc1CN[C@H]1CCCN[C@H]1c1ccccc1</smiles><smiles>O=C(O)C1NCCC[C@H]1O</smiles><smiles>O=C(CC1NCCC[C@H]1O)Cn1cnc2ccccc2c1=O</smiles><smiles>O[C@H]1CCCN2[C@@H]1[C@H](O)C[C@H]2O</smiles>

Figure 1 Biologically active 2,3-disubstituted piperidines

Because of the biomedical importance of the products, the synthesis of these $\beta$-hydroxy piperidines has attracted much attention in recent years; however, many of the synthetic approaches employ starting materials from the chi-

SYNLETT 2014, 25, 0102-0104

Advanced online publication: 12.11.2013

DOI: 10.1055/s-0033-1340074; Art ID: ST-2013-B0807-L

(C) Georg Thieme Verlag Stuttgart · New York ral pool and involve enzymatic resolution as a key reaction. ${ }^{7,8}$

We recently reported a flexible method that involves a cobalt-catalyzed hydrolytic kinetic resolution (HKR) of racemic azido epoxides with two contiguous stereocenters to generate the corresponding diols and epoxides in high optical purities $\left(97-99 \%\right.$ ee) in a single step. ${ }^{9 a}$ Here, we report a short enantioselective synthesis of two important bioactive molecules, (+)-L-733,060 (1) and (2S,3S)-3-hydroxypipecolic acid (3), based on a two-stereocenter HKR of racemic azido epoxides.

The synthesis of $(+)-\mathrm{L}-733,060$ (1; Scheme 1) commenced with the racemic azido epoxide $\mathbf{6}$, prepared from

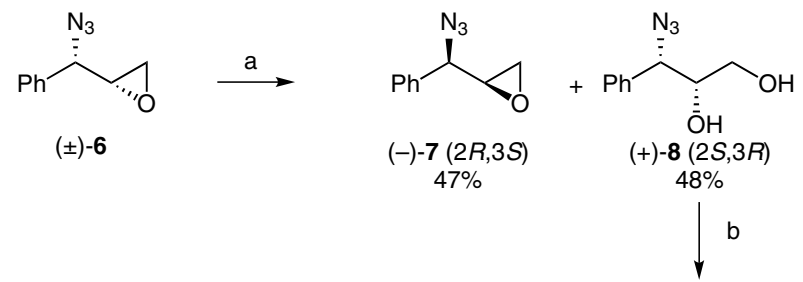

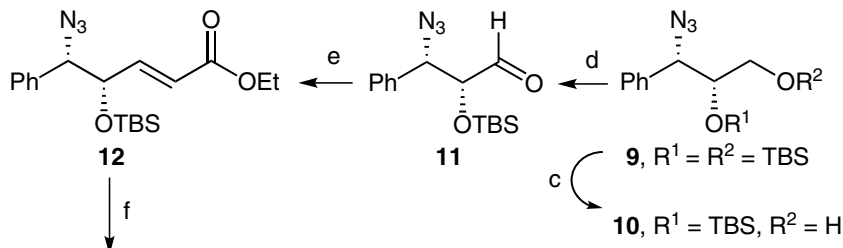<smiles>CCCN1CCC[C@@H](OCc2cc(C(F)(F)F)cc(C(F)(F)F)c2)[C@H]1c1ccccc1</smiles>

Scheme 1 Reagents and conditions: (a) (S,S)-(salen)Co(III)OAc ( $0.5 \mathrm{~mol} \%), \mathrm{H}_{2} \mathrm{O}$ ( 0.49 equiv), $0{ }^{\circ} \mathrm{C}, 14 \mathrm{~h}$; (b) TBSCl (2 equiv), imidazole, $\mathrm{CH}_{2} \mathrm{Cl}_{2}, 25^{\circ} \mathrm{C}, 12 \mathrm{~h}$; yield $98 \%$. (c) $\mathrm{CSA}, \mathrm{MeOH}, 0{ }^{\circ} \mathrm{C}, 6 \mathrm{~h}$, yield 95\%; (d) Dess-Martin periodinane, $\mathrm{CH}_{2} \mathrm{Cl}_{2}, 25^{\circ} \mathrm{C}, 1 \mathrm{~h}$, yield $98 \%$; (e) (EtO) ${ }_{2} \mathrm{POCH}_{2} \mathrm{CO}_{2} \mathrm{Et}, \mathrm{NaH}$, THF, 0 to $25^{\circ} \mathrm{C}$, $3 \mathrm{~h}$, yield $94 \%$; (f) $10 \% \mathrm{Pd} / \mathrm{C}, \mathrm{H}_{2}(1 \mathrm{~atm}), \mathrm{MeOH}, 25^{\circ} \mathrm{C}, 12 \mathrm{~h}$, then EtOH, reflux, $1 \mathrm{~h}$, yield $85 \%$; (g) TBAF, THF, $0-25^{\circ} \mathrm{C}, 2 \mathrm{~h}$, yield 96\%; (h) (i) $\mathrm{BH}_{3} \cdot \mathrm{SMe}_{2}$, THF, reflux, $10 \mathrm{~h}$; (ii) $(\mathrm{Boc})_{2} \mathrm{O}, \mathrm{Et}_{3} \mathrm{~N}$, DMAP (cat.), $\mathrm{CH}_{2} \mathrm{Cl}_{2}, 0$ to $25^{\circ} \mathrm{C}, 12 \mathrm{~h}$, yield $76 \%$ (two steps); (i) 3,5-bis(trifluoromethyl)benzyl bromide, $\mathrm{NaH}, \mathrm{DMF}, 80^{\circ} \mathrm{C}, 12 \mathrm{~h}$, yield $85 \%$; (j) TFA, $\mathrm{CH}_{2} \mathrm{Cl}_{2}, 0$ to $25^{\circ} \mathrm{C}, 18 \mathrm{~h}$, yield $89 \%$. 
commercially available cinnamyl alcohol by our previously reported procedure. ${ }^{9 a}$ The racemic azido epoxide 6 was subjected to HKR with $(S, S)$-salen-cobalt(III) acetate complex $^{9 \mathrm{~b}}(0.5 \mathrm{~mol} \%)$ and water $(0.49$ equiv $)$, which gave the corresponding diol $8(48 \%, 98 \%$ ee) and chiral epoxide $7(47 \%)$ in high optical purity. The diol 8 was readily separated from epoxide 7 by simple flash column chromatography on silica gel.

Both free hydroxy groups in diol $\mathbf{8}$ were protected to give the disilyl ether derivative 9, which was then selectively deprotected to give the monosilyl ether $\mathbf{1 0}$ in $95 \%$ yield. Dess-Martin oxidation of $\mathbf{1 0}$ gave the crude aldehyde $\mathbf{1 1}$ in $98 \%$ yield; this underwent a Wittig-Horner reaction to give the corresponding $(E)$-azido ester 12 in 94\% yield. Intramolecular reductive cyclization of $\mathbf{1 2}$ by hydrogenation over $10 \%$ palladium/carbon gave the cis-2,3-disubstituted piperidinone $\mathbf{1 3}$ in $85 \%$ yield. Deprotection of the silyl group in $\mathbf{1 3}$ with tetrabutylammonium fluoride gave the lactam 14. Reduction of lactam 14 with borane-dimethyl sulfide in tetrahydrofuran, followed by protection of the secondary amine gave the $s y n$-amino alcohol 15 in $76 \%$ yield for the two steps. Having constructed the piperidine core with the desired syn stereochemistry, we O-alkylated amino alcohol 15 with 3,5-bis(trifluoromethyl)benzyl bromide in the presence of sodium hydride to give the protected amine 16. Finally, deprotection under acidic conditions gave L-733,060 (1) in 89\% yield (overall yield $19 \%$ from 6 in ten steps).

The synthesis of $(2 S, 3 S)$-3-hydroxypipecolic acid (3; Scheme 2) commenced from (2Z)-but-2-ene-1,4-diol, which was converted into the azido aldehyde 17 by HKR, as we previously reported..$^{9 c}$ The key intermediate 20 (Scheme 2) was readily synthesized from 17, essentially by following a similar sequence of reactions to that shown in Scheme 1. Wittig olefination and intramolecular reductive cyclization gave the trans-2,3-disubstituted piperidinone core 19 in $90 \%$ yield with an intact benzyloxy group. Reduction of piperidinones $\mathbf{1 9}$ with borane-dimethyl sulfide followed by protection in situ gave trans-piperidine derivative $\mathbf{2 0}$ in $80 \%$ yield. Hydrogenation of $\mathbf{2 0}$ over palladium/carbon in methanol at 70 psi gave the corresponding alcohol 21 in 96\% yield. Finally, oxidation of alcohol 21 with ruthenium(II) chloride and sodium periodate, ${ }^{8 f, 10}$ followed by removal of both protecting groups under acidic condition ( $6 \mathrm{M}$ aq $\mathrm{HCl}$ ), completed the synthesis of (2S,3S)-3-hydroxypipecolic acid (3; overall yield $43 \%$ from 17 in six steps). The ${ }^{1} \mathrm{H}$ and ${ }^{13} \mathrm{C}$ NMR and other spectra of (+)-L-733,060 (1) and (2S,3S)-3-hydroxypipecolic acid (3) were in complete agreement with the values reported in the literature..$^{7 \mathrm{~d}, 7 \mathrm{e}, 8 \mathrm{f}, \mathrm{o}}$

In summary, we have developed short and practical enantioselective syntheses of (+)-L-733,060 (1) and (2S,3S)-3hydroxypipecolic acid (3) with good overall yields and high optical purities (ee $\leq 99 \%$ ). The key reaction in each case was a cobalt-catalyzed HKR of a racemic azido epoxide with two stereocenters. The other operationally simple reaction sequences included a Wittig reaction and an

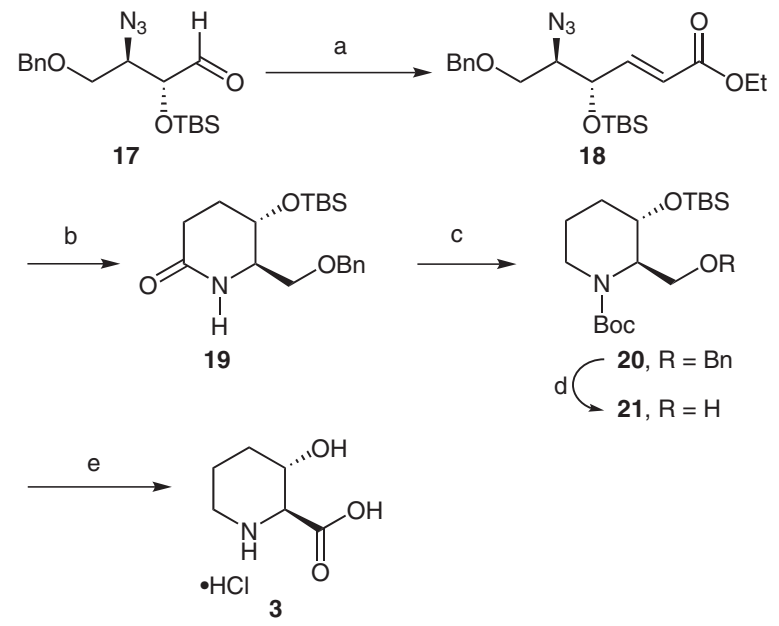

Scheme 2 Reagents and conditions: (a) (EtO) ${ }_{2} \mathrm{POCH}_{2} \mathrm{CO}_{2} \mathrm{Et}, \mathrm{NaH}$, THF, $0-25{ }^{\circ} \mathrm{C}, 1 \mathrm{~h}$, yield $93 \%$; (b) $10 \% \mathrm{Pd} / \mathrm{C}, \mathrm{H}_{2}(1 \mathrm{~atm}), \mathrm{MeOH}$, $25^{\circ} \mathrm{C}, 24 \mathrm{~h}$, yield $90 \%$; (c) $\mathrm{BH}_{3} \cdot \mathrm{SMe}_{2}$, THF, reflux, $6 \mathrm{~h}$, then $\mathrm{Na}_{2} \mathrm{CO}_{3}$, (Boc) $)_{2} \mathrm{O}, \mathrm{CH}_{2} \mathrm{Cl}_{2} / \mathrm{H}_{2} \mathrm{O}(1: 1), 25^{\circ} \mathrm{C}, 12 \mathrm{~h}$, yield $80 \%$; (d) $10 \% \mathrm{Pd} / \mathrm{C}$, $\mathrm{H}_{2}$ (70 psi), MeOH, $25^{\circ} \mathrm{C}, 24 \mathrm{~h}$, yield $96 \%$; (e) (i) $\mathrm{RuCl}_{3}(2 \mathrm{~mol} \%$ ), $\mathrm{NaIO}_{4}$ (4 equiv), $\mathrm{MeCN} / \mathrm{CCl}_{4} / \mathrm{H}_{2} \mathrm{O}$ (1:1:3), $25^{\circ} \mathrm{C}, 30 \mathrm{~min}$; (ii) $6 \mathrm{M}$ aq $\mathrm{HCl}$, reflux, $2 \mathrm{~h}$, yield $68 \%$ (two steps).

intramolecular reductive cyclization. The synthetic strategy has significant potential for further extension to other stereoisomers and related analogues of multifunctional piperidine alkaloids, owing to the flexibility available in syntheses of racemic azido epoxides with various stereochemical combinations and various substituents.

\section{Acknowledgment}

D.A.D. and P.V.C. thank CSIR, New Delhi for the award of research fellowships. The authors are also grateful to Dr. V. V. Ranade, chair of the Chemical Engineering and Process Development Division, for his constant encouragement and support.

Supporting Information for this article is available online at http://www.thieme-connect.com/ejournals/toc/synlett. Included are experimental procedures and spectral data for compounds 7-21.

\section{References}

(1) (a) Schneider, M. J. In Alkaloids: Chemical and Biological Perspectives; Vol. 10; Pelletier, S. W., Ed.; Pergamon: Oxford, 1996, 155. (b) Fodor, G. B.; Colasanti, B. In Alkaloids: Chemical and Biological Perspectives; Vol. 3; Pelletier S. W., Wiley-Interscience: New York, 1985, 1.

(c) Buffat, M. G. P. Tetrahedron 2004, 60, 1701.

(d) Laschat, S.; Dickner, T. Synthesis 2000, 1781. (e) Felpin, F.-X.; Lebreton, J. Eur. J. Org. Chem. 2003, 3693.

(f) Weintraub, P. M.; Sabol, J. S.; Kane, J. M.; Borcherding, D. R. Tetrahedron 2003, 59, 2953.

(2) (a) Baker, R.; Harrison, T.; Swain, C. J.; Williams, B. J. EP 0528495, 1993. (b) Harrison, T.; Williams, B. J.; Swain, C. J.; Ball, R. G. Bioorg. Med. Chem. Lett. 1994, 4, 2545.

(3) Desai, M. C.; Lefkwitz, S. L.; Thadeo, P. F.; Longo, K. P.; Snider, R. M. J. Med. Chem. 1992, 35, 4911.

(4) McLaughlin, N. P.; Evans, P. J. Org. Chem. 2009, 75, 518.

(5) Ferreira, F.; Greck, C.; Genet, J. P. Bull. Soc. Chim. Fr. 1997, 134, 615 . 
(6) Wijdeven, M. A.; Willemsen, J.; Rutjes, F. P. J. T. Eur. J. Org. Chem. 2010, 2831.

(7) (a) Bilke, J. L.; Moore, S. P.; O’Brien, P.; Gilday, J. Org. Lett. 2009, 11, 1935. (b) Davis, F. A.; Ramachandar, T. Tetrahedron Lett. 2008, 49, 870. (c) Liu, R.-H.; Fang, K.; Wang, B.; Xu, M.-H.; Lin, G.-Q. J. Org. Chem. 2008, 73 , 3307. (d) Emmanuvel, L.; Sudalai, A. Tetrahedron Lett. 2008, 49, 5736. (e) Cherian, S. K.; Kumar, P. Tetrahedron: Asymmetry 2007, 18, 982. (f) Oshitari, T.; Mandai, T. Synlett 2006, 3395. (g) Kandula, S. R. V.; Kumar, P. Tetrahedron: Asymmetry 2005, 16, 3579. (h) Yoon, Y.-J.; Joo, J.-E.; Lee, K.-Y.; Kim, Y.-H.; Oh, C.-Y.; Ham, W.-H. Tetrahedron Lett. 2005, 46, 739. (i) Huang, P.-Q.; Liu, L.-X.; Wei, B.-G.; Ruan, Y.-P. Org. Lett. 2003, 5, 1927. (j) Bhaskar, G.; Rao, B. V. Tetrahedron Lett. 2003, 44, 915. (k) Takahashi, K.; Nakano, H.; Fijita, R. Tetrahedron Lett. 2005, 46, 8927. (l) Liu, L.-. X.; Ruan, Y.-P.; Guo, Z.-Q.; Huang, P.-Q. J. Org. Chem. 2004, 69, 6001. (m) Lemire, A.; Grenon, M.; Pourashraf, M.; Charette, A. B. Org. Lett. 2004, 6, 3517. (n) Prevost, S.; Phansavath, P.; Haddad, M. Tetrahedron: Asymmetry 2010, 21, 16. (o) Kumaraswamy, G.; Pitchaiah, A. Tetrahedron 2011, 67, 2536. (p) Garrido, N. M.; García, M.; Sánchez, R.; Díez, D.; Urones, J. Synlett 2010, 387. (q) Mizuta, S.; Onomura, O. RSC Adv. 2012, 2, 2266. (r) Pansare, S. V.; Paul, E. K. Org. Biomol. Chem. 2012, 10, 2119. (s) Tsai, M.-R.; Chen, B.-F.; Cheng, C.-C.; Chang, N.-C. J. Org. Chem. 2005, 70, 1780.

(8) (a) Chattopadhyay, S. K.; Roy, S. P.; Saha, T. Synthesis 2011, 2664. (b) Lemire, A.; Charette, A. B. J. Org. Chem. 2010, 75, 2077. (c) Chiou, W. H.; Lin, G. H.; Liang, C. W. J. Org. Chem. 2010, 75, 1748. (d) Chung, H. S.; Shin, W. K.; Choi, S. Y.; Chung, Y. K.; Lee, E. Tetrahedron Lett. 2010, 51, 707. (e) Yoshimura, Y.; Ohara, C.; Miyagawa, T.; Takahata, H. Heterocycles 2009, 77, 635. (f) Wang, B.; RunHua, L. Eur. J. Org. Chem. 2009, 2845. (g) Kumar, P. S.; Baskaran, S. Tetrahedron Lett. 2009, 50, 3489. (h) Cochi, A.; Burger, B.; Navarro, C.; Pardo, D. G.; Cossy, J.; Zhao, Y.; Cohen, T. Synlett 2009, 2157. (i) Yoshimura, Y.; Ohara, C.; Imahori, T.; Saito, Y.; Kato, A.; Miyauchi, S.; Adachi, I.; Takahata, H. Bioorg. Med. Chem. 2008, 16, 8273. (j) Pham, V.-T.; Joo, J.-E.; Tian, Y.-S.; Chung, Y.-S.; Lee, K.-Y.; Oh, C.-Y.; Ham, W.-H. Tetrahedron: Asymmetry 2008, 19, 318. (k) Ohara, C.; Takahashi, R.; Miyagawa, T.; Yoshimura, Y.; Kato, A.; Adachi, I.; Takahata, H. Bioorg. Med. Chem. Lett. 2008, 18, 1810. (1) Liu, L.-X.; Peng, Q.-L.; Huang, P.-Q. Tetrahedron: Asymmetry 2008, 19, 1200. (m) Alegret, C.; Ginesta, X.; Riera, A. Eur. J. Org. Chem. 2008, 1789.

(n) Chavan, S. P.; Harale, K. R.; Dumare, N. B.; Kalkote, U. R. Tetrahedron: Asymmetry 2011, 22, 587. (o) Chavan, S. P.; Dumare, N. B.; Harale, K. R.; Kalkote, U. R. Tetrahedron Lett. 2011, 52, 404. (p) Chavan, S. P.; Harale, K.; Pawar, K. P. Tetrahedron Lett. 2013, 54, 4851. (q) Jourdant, A.; Zhu, J. Tetrahedron Lett. 2000, 41, 7033. (r) Kumar, P.; Bodas, M. S. J. Org. Chem. 2005, 70, 360. (s) Kalamkar, N. B.; Kasture, V. M.; Dhavale, D. D. J. Org. Chem. 2008, 73, 3619. (t) Kokatla, H. P.; Lahiri, R.; Kancharla, P. K.; Doddi, V. R.; Vankar, Y. D. J. Org. Chem. 2010, 75, 4608. (u) Liang, N.; Datta, A. J. Org. Chem. 2005, 70, 10182. (v) Kim, I. S.; Oh, J. S.; Zee, O. P.; Jung, Y. H. Tetrahedron 2007, 63, 2622. (w) Bodas, M. S.; Kumar, P. Tetrahedron Lett. 2004, 45, 8461.

(9) (a) Reddy, R. S.; Chouthaiwale, P. V.; Suryavanshi, G.; Chavan, V. B.; Sudalai, A. Chem. Commun. 2010, 46, 5012. (b) Tokunaga, M.; Larrow, J. F.; Kakiuchi, F.; Jacobsen, E.
N. Science 1997, 277, 936. (c) Devalankar, D. A.; Sudalai, A. Tetrahedron Lett. 2012, 53, 3213.

(10) (a) Nunez, M. T.; Martin, V. S. J. Org. Chem. 1990, 55, 1928. (b) Carlsen, P. H. J.; Katsuki, T.; Martin, V. S.; Sharpless, K. B. J. Org. Chem. 1981, 46, 3936.

(11) Hydrolytic Kinetic Resolution of Azido Epoxide 6 $\mathrm{AcOH}(0.014 \mathrm{~g}, 0.24 \mathrm{mmol})$ was added to a solution of $(S, S)$ (salen)Co(II) complex (0.024 mmol, $0.5 \mathrm{~mol} \%)$ in toluene (1 $\mathrm{mL}$ ), and the mixture was stirred at $25^{\circ} \mathrm{C}$ in open air for 30 $\mathrm{min}$. During this time the color changed from orange-red to a dark brown. The solution was then concentrated under reduced pressure to give the $\mathrm{Co}(\mathrm{III})-$ salen complex as a brown solid. To this were added the racemic azido epoxide $6(0.84 \mathrm{~g}, 4.85 \mathrm{mmol})$ and $\mathrm{H}_{2} \mathrm{O}(0.043 \mathrm{~g}, 2.42 \mathrm{mmol})$ at $0{ }^{\circ} \mathrm{C}$, and the resulting mixture was stirred at $0{ }^{\circ} \mathrm{C}$ for $14 \mathrm{~h}$. When the reaction was complete (TLC), the crude product was purified by column chromatography [silica gel, PE-EtOAc] to give chiral azido epoxide 7 (9:1 PE-EtOAc) and the chiral azido diol 8 (1:1 PE-EtOAc) in pure form.

(2R,3S)-3-Azido-3-phenylpropane-1,2-diol (8)

Yellow liquid; yield: $450 \mathrm{mg}(48 \%, 98 \%$ ee $) ;[\alpha]_{\mathrm{D}}{ }^{25}+188(c$ $\left.1, \mathrm{CHCl}_{3}\right)$ (lit. ${ }^{9 \mathrm{a}}-188$ for the antipode). IR $\left(\mathrm{CHCl}_{3}\right): 1602$, 2099, 2932, 3052, 3392 (br) cm ${ }^{-1} .{ }^{1} \mathrm{H}$ NMR (200 MHz, $\left.\mathrm{CDCl}_{3}\right): \delta=3.30(\mathrm{dd}, J=11.5,6.0 \mathrm{~Hz}, 1 \mathrm{H}), 3.44(\mathrm{~d}$, $J=11.5 \mathrm{~Hz}, 1 \mathrm{H}), 3.80$ (br s, $1 \mathrm{H}), 3.62-3.94(\mathrm{~m}, 1 \mathrm{H}), 4.52$ $(\mathrm{d}, J=8.1,1 \mathrm{H}), 7.28-7.35(\mathrm{~m}, 5 \mathrm{H}) .{ }^{13} \mathrm{C}$ NMR $(50 \mathrm{MHz}$, $\left.\mathrm{CDCl}_{3}\right): \delta=2.8,68.1,75.0,127.5,128.7,128.9,136.2$. Anal. Calcd for $\mathrm{C}_{9} \mathrm{H}_{11} \mathrm{~N}_{3} \mathrm{O}_{2}: \mathrm{C}, 55.95 ; \mathrm{H}, 5.74 ; \mathrm{N}, 21.75$. Found: C, 56.10; H, 5.65; N, 21.60; HPLC: Chiral OD-H column, hexane $-i-\mathrm{PrOH}(90: 10,0.5 \mathrm{~mL} / \mathrm{min}), 254 \mathrm{~nm}$; $t_{\mathrm{R}(\text { major })}=14.84 \mathrm{~min}, t_{\mathrm{R}(\text { minor })}=15.57 \mathrm{~min}$.

(2S)-2-[(R)-Azido(phenyl)methyl]oxirane (7)

Yellow liquid; yield: $400 \mathrm{mg}(47 \%)$; $[\alpha]_{\mathrm{D}}{ }^{25}-120$ (c 1, $\left.\mathrm{CHCl}_{3}\right)\left(\right.$ lit. ${ }^{9 \mathrm{a}}+120$ for the antipode). IR $\left(\mathrm{CHCl}_{3}\right): 2105$, $2932,3025 \mathrm{~cm}^{-1}$. ${ }^{1} \mathrm{H}$ NMR $\left(200 \mathrm{MHz}, \mathrm{CDCl}_{3}\right): \delta=2.73-$ $2.84(\mathrm{~m}, 2 \mathrm{H}), 3.23-3.29(\mathrm{~m}, 1 \mathrm{H}), 4.25(\mathrm{~d}, J=6.1,1 \mathrm{H})$, 7.35-7.47 (m, $5 \mathrm{H}) .{ }^{13} \mathrm{C}$ NMR $\left(50 \mathrm{MHz}, \mathrm{CDCl}_{3}\right): \delta=44.6$, 54.6, 66.8, 127.2, 128.8, 128.9, 135.7. Anal. Calcd for $\mathrm{C}_{9} \mathrm{H}_{9} \mathrm{~N}_{3} \mathrm{O}: \mathrm{C}, 61.70 ; \mathrm{H}, 5.18 ; \mathrm{N}, 23.99$. Found: $\mathrm{C}, 61.79 ; \mathrm{H}$, $5.14 ; \mathrm{N}, 23.90$

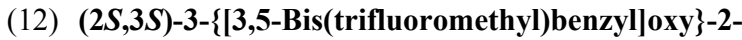
phenylpiperidine $[1 ;(+)-\mathrm{L}-\mathbf{7 3 3 , 0 6 0}]$

Colorless oil; yield: $110 \mathrm{mg}(89 \%),[\alpha]_{\mathrm{D}}{ }^{25}+35.2(c 0.66$, $\left.\mathrm{CHCl}_{3}\right)\left\{\right.$ lit. $^{7 \mathrm{j}}+34.29\left(\right.$ c $\left.\left.1.32, \mathrm{CHCl}_{3}\right)\right\}$. IR (neat): 1277 , $1370,2950 \mathrm{~cm}^{-1} .{ }^{1} \mathrm{H}$ NMR $\left(\mathrm{CDCl}_{3}, 200 \mathrm{MHz}\right): \delta=1.40$ $2.04(\mathrm{~m}, 3 \mathrm{H}), 2.22$ (br d, $J=13 \mathrm{~Hz}, 1 \mathrm{H}), 2.60$ (s, $1 \mathrm{H}), 2.76$ $2.81(\mathrm{~m}, 1 \mathrm{H}), 3.23-3.38(\mathrm{~m}, 1 \mathrm{H}), 3.66(\mathrm{~s}, 1 \mathrm{H}), 3.84(\mathrm{~s}, 1$ $\mathrm{H}), 4.12(\mathrm{~d}, J=12.0 \mathrm{~Hz}, 1 \mathrm{H}), 4.54(\mathrm{~d}, J=12.2 \mathrm{~Hz}, 1 \mathrm{H})$, $7.20-7.50(\mathrm{~m}, 7 \mathrm{H}), 7.78(\mathrm{~s}, 1 \mathrm{H}) .{ }^{13} \mathrm{C} \mathrm{NMR}\left(\mathrm{CDCl}_{3}, 50\right.$ MHz): 20.6, 27.5, 47.1, 64.0, 70.5, 77.2, 120.9, 124.1, 127.7, 128.5, 128.7, 128.9, 131.2, 141.6, 142.3. Anal. Calcd for $\mathrm{C}_{20} \mathrm{H}_{19} \mathrm{~F}_{6} \mathrm{NO}: \mathrm{C}, 59.55 ; \mathrm{H}, 4.75 ; \mathrm{N}, 3.47$. Found: $\mathrm{C}, 59.52 ; \mathrm{H}$, $4.81 ; \mathrm{N}, 3.56$.

(2S,3S)-3-Hydroxypiperidine-2-carboxylic Acid [3; (2S,3S)-3-Hydroxypipecolic Acid] Colorless solid; yield: $20 \mathrm{mg}(68 \%) ; \mathrm{mp} 232{ }^{\circ} \mathrm{C} ;[\alpha]_{\mathrm{D}}{ }^{25}$ $+14.2\left(c 1, \mathrm{H}_{2} \mathrm{O}\right)\left\{\right.$ lit. $\left.^{8 \mathrm{f}}[\alpha]_{\mathrm{D}}{ }^{23}+14.5\left(c 0.4, \mathrm{H}_{2} \mathrm{O}\right)\right\}$. IR (neat): $1685,3420 \mathrm{~cm}^{-1}$. ${ }^{1} \mathrm{H}$ NMR $\left(200 \mathrm{MHz}, \mathrm{D}_{2} \mathrm{O}\right): \delta=1.62-1.80$ (m, $2 \mathrm{H}), 2.00-2.08$ (m, $2 \mathrm{H}), 3.10$ (s, $1 \mathrm{H}), 3.32-3.39$ (m, 1 $\mathrm{H}), 3.80(\mathrm{~d}, J=7.6 \mathrm{~Hz}, 1 \mathrm{H}), 4.10-4.17(\mathrm{~m}, 1 \mathrm{H}) .{ }^{13} \mathrm{C} \mathrm{NMR}$ $\left(50 \mathrm{MHz}, \mathrm{D}_{2} \mathrm{O}\right): \delta=20.0,30.1,43.9,62.5,65.9,171.3$. Anal. Calcd for $\mathrm{C}_{6} \mathrm{H}_{11} \mathrm{NO}_{3}: \mathrm{C}, 49.65 ; \mathrm{H}, 7.64 ; \mathrm{N}, 9.65$. Found: $\mathrm{C}$, 49.60; H, 7.69; N, 9.70. 